\title{
Improved photostability of Vitamin A palmitate originating from self-assembled supramolecular gels
}

\author{
WANG Hong, FANG Fang, LI Xue, FU Cheng \& YANG YaJiang* \\ School of Chemistry and Chemical Engineering, Huazhong University of Science and Technology, Wuhan 430074, China
}

Received March 2, 2012; accepted May 3, 2012; published online September 14, 2012

\begin{abstract}
Retinyl palmitate (RP) is a derivative of Vitamin A and widely applied as the active component in the fields of cosmetic and pharmaceutics. RP can easily lose its physiological activity under UV irradiation due to its photosensitivity. In this work, it was found that the activity of RP remained $75 \%$ after 80 min of UVA irradiation when RP was entrapped in supramolecular gels formed by self-assembly of sorbitol-based gelators. By contrast, the activity of RP in conventional hydroxyethyl cellulose gels only remained $10 \%$ under the same conditions. These results showed that the supramolecular gels exhibited a significant protective effect on the photostability of RP. UV spectra of RP in supramolecular gels and corresponding solutions showed no distinct differences, indicating no change of the physicochemical properties of RP. The images of field-emission scanning electron microscopy and fluorescent optical microscopy suggested that the protection by supramolecular gels on the photostability of RP may be attributed to the three-dimensional network structure formed by the self-assembly of the gelators, which reduced the molecular collisions and the degradation process of the photoactivated RP. The present results showed that this protection can be influenced by the structures and the concentration of gelators as well as by the solvent composition.
\end{abstract}

supramolecular gels, self-assembly, retinyl palmitate, photostability

Citation: Wang H, Fang F, Li X, et al. Improved photostability of Vitamin A palmitate originating from self-assembled supramolecular gels. Chin Sci Bull, 2012, 57: 4257-4263, doi: 10.1007/s11434-012-5451-1

Vitamin A (retinol) is an essential human nutrient that plays an important role in critical physiological functions including vision and epidermal cell growth [1]. Retinyl esters, particularly retinyl palmitate (denoted as RP, Scheme 1), are widely applied in many products because they are more chemically stable than retinol. However, the use of RP as epidermal medicines is limited due to its significant photosensitivity, easily losing its physiological activity under UV irradiation. Therefore, the protection of RP towards photodecomposition has been the subject of increasing attention. Various photostability agents and inclusion agents are currently used to protect photodegradation of RP $[2,3]$.

Manabu et al. [4] developed an eye drop containing RP, in which, polyoxyethylene-polyoxypropylene glycols were used as inclusion agents for treatment of corneal and conjunctival injuries. RP retention is approximately $85 \%$ after

*Corresponding author (email: yjyang@ mail.hust.edu.cn) storage for two months. Semenzato et al. [5] prepared O/W cosmetic emulsions with high viscoelastic properties as carrier of RP. Results showed that the chemical stability of RP strictly depends on the physical stability of the formulation and in particular on the presence of a coherent gel-like structure in the external phase of the emulsion. Young et al. [6] investigated photodecomposition of retinol entrapped within liposomes in the presence of $\alpha$-tocopherol and the oxybenzone. It was found that the actual entrapment of these protecting species in the liposomes is important in enhancing the stability of the drug. Carlotti et al. [7] employed solid lipid nanoparticles (SLNs) dispersed in O/W emulsions as carriers for RP and found that the SLNs themselves show physical UV-blocking action. SLNs in O/W emulsions protect RP from photodegradation induced by UVA and UVB radiation, probably due to the light-scattering properties of SLNs. Carlotti et al. [8] also employed hydroxylethyl cellulose gels to enhance the photostability of 
$\mathrm{RP}$ and investigated the effect of the $\mathrm{pH}$ of the gels on the photostability of RP. It was found that RP in the gels at $\mathrm{pH}$ 4 and 8 was less stable than at $\mathrm{pH} 5.6$ and 7. Undegraded $\mathrm{RP}$ at $\mathrm{pH} 7$ remained approximately $20 \%$ under UVA radiation for $90 \mathrm{~min}$. Cyclodextrins (CD) are useful inclusion agents for the protection of RP. Sapino et al. [9] employed two synthetic alkyl carbonates of $\gamma-\mathrm{CD}$ derivatives in hydroxylethyl cellulose gels as inclusion agent of retinol. The results of a permeation study indicated that the complexation with alkyl- $\gamma-C D$ increases the amount of vitamin A that accumulates into the skin improving its effectiveness. Retinol retention was approximately $80 \%$ under UVB radiation for $30 \mathrm{~min}$.

Supramolecular gels are semisolid materials formed by self-assembly of low-molecular mass gelators in organic or aqueous solvents via noncovalent interaction [10-12]. The preparation process is simple and more feasible in comparison with the different carriers described above, such as O/W emulsions, liposomes and SLNs. Supramolecular gels consisting of biocompatible components have a great potential application in the fields of topical medicines and cosmetics [13]. In addition, we speculated that the threedimensional network structure formed by the gelator aggregates in supramolecular gels may be beneficial to preserve the photostability of RP. Therefore we propose a new strategy for protecting the photostability of RP in this work. To the best of our knowledge, the use of the supramolecular gels to protect the photostability of RP has not been reported previously.

In the present work, supramolecular gels were prepared by self-assembly of a series of sorbitol-based gelators including 1,3:2,4-di- $O$-benzylidene- $D$-sorbitol (DBS), 1,3:2,4-di- $O$ $p$-methylbenzylidene- $D$-sorbitol (MDBS) and 1,3:2,4-di- $O$ $m, p$-dimethylbenzylidene- $D$-sorbitol (DMDBS) (Scheme 1). Low-molecular-weight PEG or 1,2-propylene glycol and ethanol were used as matrices of the supramolecular gels. The mechanism of the protection effect excercised by the supramolecular gels on the photostability of RP under

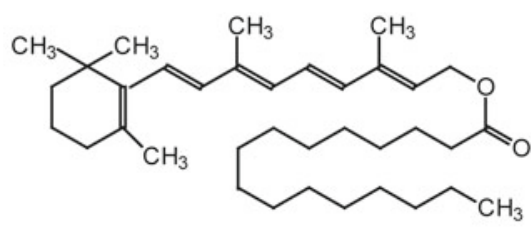

RP

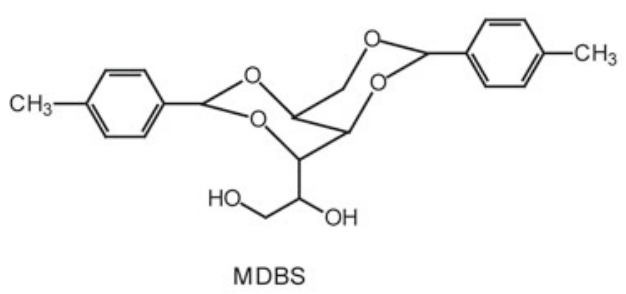

various conditions was investigated by field-emission scanning electron microscopy, UV spectroscopy and fluorescent optical microscopy.

\section{Experimental}

\subsection{Materials}

1,3:2,4-di- $O$-benzylidene- $D$-sorbitol (DBS, purity 99\%), 1,3:2,4-di- $O$ - $p$-methylbenzylidene- $D$-sorbitol (MDBS, purity $99 \%)$ and 1,3:2,4-di- $O-m, p$-dimethylbenzylidene- $D$ sorbitol (DMDBS, purity 99\%) were commercially available (Hubei Huabang Chemicals Co). All-trans-retinyl palmitate (RP, purity $99.9 \%, \geqslant 1700000 \mathrm{IU} / \mathrm{g}$ ) was purchased from Xiamen Kingdomway Co. The appropriate amount of $\mathrm{RP}$ was dissolved in ethanol to prepare the stock solutions of RP $\left(9 \times 10^{-4} \mathrm{~mol} / \mathrm{L}\right)$. Polyethylene glycol 400 (PEG400), 1,2-propylene glycol and ethanol were of analytical grade and used as received. Hydroxyethyl cellulose (HEC, 38000 $\mathrm{mPa} \mathrm{s}$ at $25^{\circ} \mathrm{C}$ ) was purchased from Shandong Yutian Chemicals Co. Ultrapure water was produced by a Millipore Direct-Q system.

\subsection{Preparation of supramolecular gels containing RP}

A weighed amount of DBS, MDBS and DMDBS were separately added to a mixture of 1,2-propylene glycol/ethanol or PEG400/ethanol. The mixture was stirred and heated in a water bath until the solid completely dissolved and then allowed to cool to $30^{\circ} \mathrm{C}$. Subsequently an appropriate amount of a stock solution of RP was added. The ratio of 1,2-propylene glycol/ethanol and PEG400/ethanol, as well as the concentration of RP and gelators in the mixture will be described in the corresponding Figures. The mixture was subsequently allowed to cool to room temperature to form the supramolecular gels (designated as DBS gel, MDBS gel and DMDBS gel). The corresponding solutions of 1,2propylene glycol/ethanol and PEG400/ethanol without the

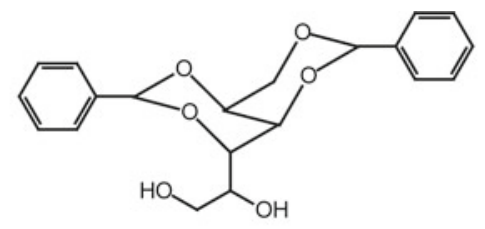

DBS

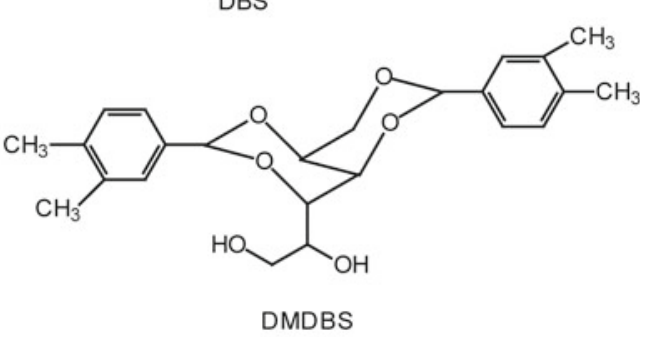

Scheme 1 Chemical structures of RP, DBS, MDBS and DMDBS. 
gelators were prepared as the reference.

\subsection{Photodegradation of RP}

The gels and corresponding solutions containing RP in 5 mm quartz cuvettes were irradiated with UVA light (CLEO Performance $40 \mathrm{~W}$, Philips). The samples were taken from the cuvette with designed intintervals $(20 \mathrm{~min})$ and diluted with ethanol to the appropriate concentration in the test tube. The absorbance at $327 \mathrm{~nm}$, the characteristic absorption of $\mathrm{RP}$, was recorded using a UV-Vis spectrophotometer (TU-1810, Beijing Pushi General Co.). The recovery of RP was found to be approximately $99 \%(n=3, \mathrm{RSD}=1.78 \%)$. The retained activity (RA) was used to estimate the photostability of RP. The retained activity is defined as follows:

$$
\mathrm{RA}=\frac{A}{A_{0}} \times 100 \% \text {. }
$$

Herein $A_{0}$ is the absorbance of RP in the sample before UVA irradiation and $A$ is the absorbance of RP in the sample after UVA irradiation.

Similarly, UV spectra of the gels and corresponding solutions containing RP in $2 \mathrm{~mm}$ quartz cuvettes were recorded employing a UV-Vis spectrophotometer. The gels and corresponding solutions without RP were used as references $[6,7]$.

\subsection{Fluorescent optical microscopy (FOM) and field- emission scanning electron microscopey (FE-SEM)}

The hot solution mixtures described in section 1.2 were dropped on a preheated glass plate. After cooling to room temperature, the gel samples were imaged by FOM (IX71, Olympus). Similarly, the morphological analysis of the gel samples with or without RP was performed by FE-SEM (Sirion 200, FEI). The gel samples were frozen in liquid nitrogen and then freeze-dried (Freezone 6, Labconco). All samples were coated with Au. The accelerating voltage was $10 \mathrm{kV}$.

\subsection{Preparation of a conventional hydroxyethyl cellu- lose gel containing RP}

The conventional hydroxyethyl cellulose (HEC) gel was prepared in accordance with the method described previously [8]. Briefly, a mixture of $0.2 \mathrm{wt} \%$ of RP (approximately $\left.3.8 \times 10^{-3} \mathrm{~mol} / \mathrm{L}\right), 2 \mathrm{~g}$ of $\mathrm{HEC}$ and $98 \mathrm{~mL}$ of water was stirred at $25^{\circ} \mathrm{C}$ for $2 \mathrm{~min}$.

\section{Results and discussion}

\subsection{Effect of gelator structures and concentrations on the photostability of RP}

DBS, MDBS and DMDBS are efficient gelators to form supramolecular gels in organic solvents [14-16]. In our experiments, it was found that a mixture of 1,2-propylene glycol/ethanol (1/2, v/v) or PEG400/ethanol (1/1-3, v/v) can transform to a stable and transparent gel in the presence of these sorbitol-based gelators. The minimum gelation concentration of the gelators was found to be approximately $0.4 \mathrm{wt} \%$ in both solvent systems. In other words, the three-dimensional network of the supramolecular structures can be formed through self-assembly of the sorbitol-based gelators in the solvents when their concentration is more than $0.4 \mathrm{wt} \%$. The solvent molecules are immobilized in the three-dimensional networks by the capillary effect. The nanostructure of the three-dimensional network can be used as a molecular platform to load the guest molecules. Due to a very low concentration of RP $\left(6 \times 10^{-4} \mathrm{~mol} / \mathrm{L}\right)$ in the gels, the existence of RP has no influence on the formation of the supramoleuclar gels. As reported previously, the guest molecules in the network exhibit different properties from those in the homogeneous solution [17-19]. Figure 1 shows the photostability of RP in supramolecular gels formed by self-assembly of DBS, MDBS and DMDBS in propylene glycol/ethanol (Figure 1(a)). The photostability of RP in the corresponding solutions was also measured as the control. Figure 1(b) shows the effect of MDBS concentration in the
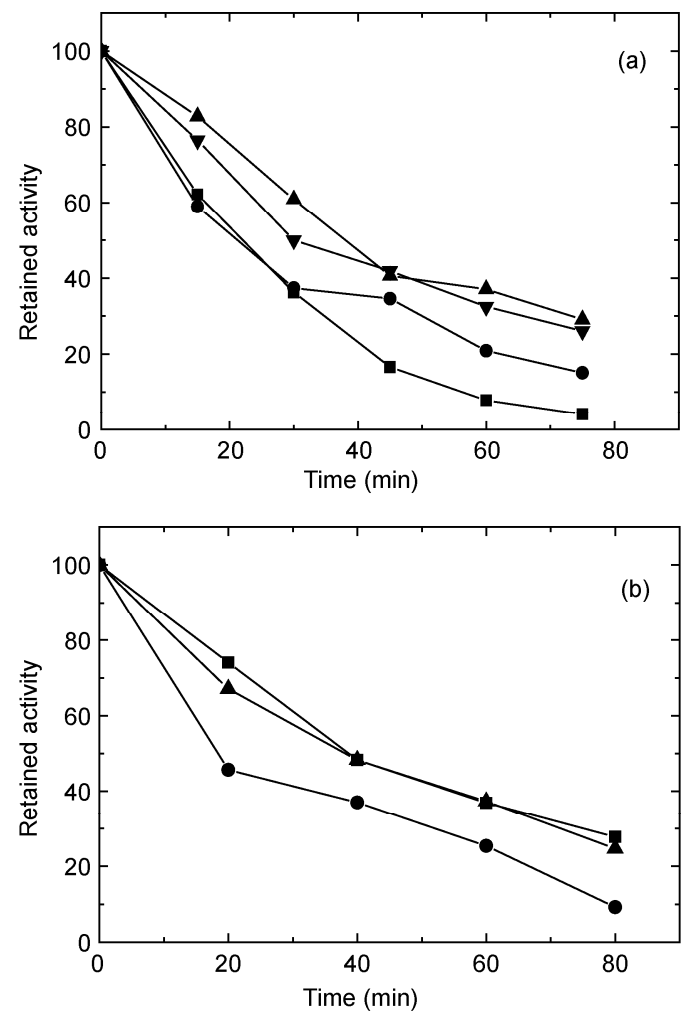

Figure 1 (a) Effect of gelator structures on the photostability of RP in

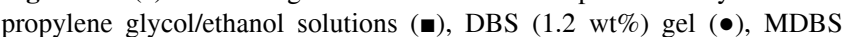
$(0.6 \mathrm{wt} \%)$ gel $(\boldsymbol{\Delta})$ and DMDBS (0.6 wt $\%)$ gel ( $)$. (b) Effect of MDBS concentrations on the photostability of RP in MDBS gels. $0.05 \mathrm{wt} \%(\bullet)$, $0.4 \mathrm{wt} \%(\boldsymbol{\Delta})$ and $0.8 \mathrm{wt} \%(\boldsymbol{\bullet})$. The concentration of RP in all samples was $6 \times 10^{-4} \mathrm{~mol} / \mathrm{L}$. The ratio of propylene glycol/ethanol was $1 / 2(\mathrm{v} / \mathrm{v})$. 
gels on the photostability of RP. We note that the aim of this work is to preliminarily estimate photostability of RP in supramolecular gels. Thus, only UVA light was employed in all irradiation measurements.

As shown in Figure 1(a), the activity of RP in MDBS gels remained $30 \%$ after irradiation of UVA for $75 \mathrm{~min}$, which is higher than that in the corresponding solution (4\%). It suggested that the photostability of RP entrapped in the gel was efficiently protected in comparison with that in corresponding solutions. Similar results can be observed in the case of DBS and DMDBS gels. However, the retained activity of RP in DBS gels is lower than that in MDBS and DMDBS gels. This can be attributed to the difference of their chemical structures. As shown in Scheme 1, MDBS and DMDBS have one and two extra methyl groups in comparison DBS. Thus the microenvironment of threedimensional network formed by MDBS and DMDBS is facilitated to entrap the hydrophobic RP molecules. Therefore, MDBS gels were used in successive experiments to investigate the photostability of RP in gels.

In general, three-dimensional network structures in the supramolecular gels greatly depend upon the concentration of the gelator [20,21]. Figure 1(b) shows the effect of the concentrations of MDBS on the photostability of RP. As mentioned above, the minimum gelation concentration of MDBS in propylene glycol/ethanol is approximately 0.4 $\mathrm{wt} \%$. In the case of propylene glycol/ethanol containing $0.05 \mathrm{wt} \%$ of MDBS (no gel state), the retained activity of RP was found to be $9 \%$ after 80 min of UVA irradiation. By contrast, the retained activity of RP in the gel formed by 0.4 wt $\%$ of MDBS increased to $30 \%$. We note that the improvement caused by higher concentrations of MDBS is limited. A small effect on the retained activity of RP was found when $0.8 \mathrm{wt} \%$ of MDBS was employed. Since the concentration of RP in MDBS gel was fixed, this result implies that there is a proper matching concentration relationship between RP and gelator. The excess of MDBS exerts no influence on the increase of the photostability of RP. This will be further discussed below.

\subsection{Effect of solvent composition of the supramolecular gels on the photostability of RP}

The main component in the supramolecular gel is solvent. The solvent composition greatly affects the formation and property of the gels $[22,23]$. Two kinds of solvent systems for the MDBS gels were investigated in this work. One of them is the low molecular weight propylene glycol/ethanol $(1 / 2, v / v)$ and the other is oligomeric PEG400/ethanol $(1 / 1-3, v / v)$. Figure 2 shows the retained activity of RP in two kinds of supramolecular gels after irradiation with UVA. As shown in Figure 2(a), there seems to be no significant effects on the protection of RP in both gels. The protection of gel formed in oligomeric PEG400/ethanol is slightly better than that of the gel formed in propylene glycol/ethanol.
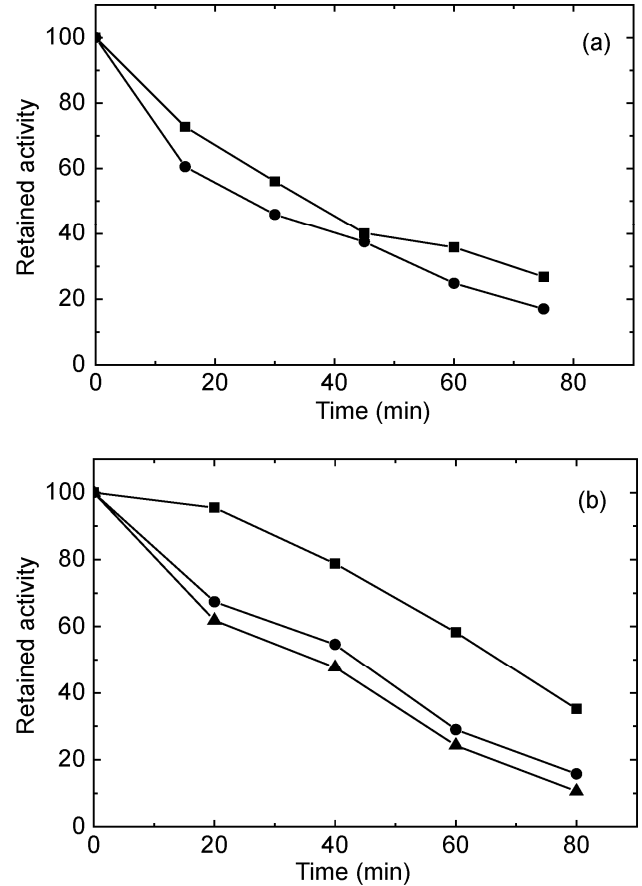

Figure 2 (a) Effect of solvent composition on the photostability of RP in propylene glycol/ethanol $(1 / 2, \mathrm{v} / \mathrm{v})(\bullet)$ and PEG400/ethanol (1/2, v/v) (•). Concentrations of MDBS in all samples were $0.4 \mathrm{wt} \%$. (b) Effect of solvent composition on the photostability of RP in PEG400/ethanol (1/1, v/v) $(\bullet)$, PEG400/ethanol $(1 / 2, \mathrm{v} / \mathrm{v})(\bullet)$ and PEG400/ethanol $(1 / 3, \mathrm{v} / \mathrm{v})(\boldsymbol{\Lambda})$. Concentrations of MDBS in all samples were $0.6 \mathrm{wt} \%$. Concentrations of $\mathrm{RP}$ in all samples were $6 \times 10^{-4} \mathrm{~mol} / \mathrm{L}$.

This may be attributed to a fact that the entangled long chains of PEG are more beneficial to entrap RP molecules. Thus, the effect of different ratio of PEG400/ethanol on the photostability of RP was investigated (Figure 2(b)). It was found that the retained activity of RP decreased with a decrease of the PEG content, indicating that the long chains of PEG also retard the photodegradation of RP. This conclusion is consistent with the protection effect of PEG on photosensitive hemin, reported by Inamura et al. [24]. They found that the photodegradation rate of the PEG/hemin complex was only half of the photodegradation rate of hemin in DMSO.

\subsection{Effect of RP concentrations in MDBS gels on the photostability of RP}

As discussed in Section 2.1, we suggest that there is a proper matching concentration relationship between MDBS and RP in gels. Figure 3 shows the effect of RP concentration in the MDBS gels on the photostability of RP. The values on the $Y$ axis are the difference of retained activity of RP between MDBS gels and corresponding solutions. As shown in Figure 3, the retained activity of RP is the lowest at the highest concentration of RP $\left(10^{-2} \mathrm{~mol} / \mathrm{L}\right)$. It indicates that excessive RP is not protected because of the constant content of MDBS, resulting in the low retained activity. The 


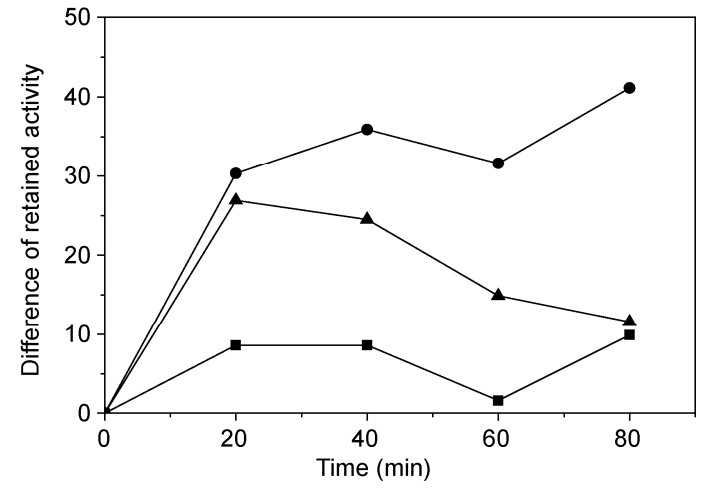

Figure 3 Effect of different RP concentrations on the difference of retained activity between MDBS gels and corresponding solutions. Concentrations of RP were $1 \times 10^{-2} \mathrm{~mol} / \mathrm{L}(\bullet), 2.5 \times 10^{-3} \mathrm{~mol} / \mathrm{L}(\bullet)$ and $2 \times 10^{-4}$ $\mathrm{mol} / \mathrm{L}(\boldsymbol{\Delta})$. Concentration of MDBS in PEG400/ethanol $(1 / 2, \mathrm{v} / \mathrm{v})$ was 0.6 $\mathrm{wt} \%$.

retained activity of RP was also not high in case a low concentrations of RP was employed $\left(10^{-4} \mathrm{~mol} / \mathrm{L}\right)$. In our experiments, it was found that the color of the system was nearly colorless and transparent when $10^{-4} \mathrm{~mol} / \mathrm{L}$ of RP was used. In this case, the transmission of the UV light increased, resulting in an increase of the photodegradation of RP. The retained activity of RP showed the highest value when the $\mathrm{RP}$ concentration was $10^{-3} \mathrm{~mol} / \mathrm{L}$. It implies that this concentration is basically matching the inclusion effect of MDBS gels.

\subsection{Comparison of MDBS gels and conventional poly- meric HEC gels}

The hydroxyethyl cellulose (HEC) gel is widely used as a drug carrier. The protection effect of a HEC gel on the photostability of RP has also been reported elsewhere [6]. The polymeric HEC gel is completely different from the supramolecular gel both in structure and in properties. Figure 4(a) shows a comparison of the protection effects of the two kinds of gels on the photostability of RP. The retained ac- tivity of RP was found to be approximately $75 \%$ in the MDBS gel, and only $10 \%$ in the HEC gel after 80 min of UVA irradiation. In addition, it was also found that the HEC gel formed by $2 \mathrm{wt} \%$ of HEC and $98 \mathrm{wt} \%$ of water is opaque and the MDBS gel is transparent (Figure 4(b)). Since it is well known that UV light can transmit a transparent gel more easily, the photodegradation of RP is understandable. However, the results in Figure 4(b) suggest that the protection effect of the MDBS gel on the photostability of RP is much better than that of the HEC gel. This may be of great significance for the preparation of cosmetics containing RP.

\subsection{Mechanism of the protective effect of MDBS gels on the photostability of RP}

The above discussion indicates that the supramolecular gel has an obvious protective effect on the photostability of RP. The mechanism of the protective effect may involve the particular microenvironment of RP located in the MDBS gel and the microstructure of the MDBS gel. Figure 5(a) and (b) shows FE-SEM images of MDBS xerogels formed in propylene glycol/ethanol $(1 / 2, \mathrm{v} / \mathrm{v})$ in the presence of RP and in the absence of RP, respectively. Three-dimensional network structures consisting of fibril bundles can be clearly observed, originating from the self-assembly of MDBS in propylene glycol/ethanol. The diameter of the fibril aggregates is approximately $100 \mathrm{~nm}$. The presence of RP in the gels seems to have no influence on the formation of the three-dimensional network structures. A lot of solvent "chambers", as a location of RP, are present in the threedimensional network. RP molecules are entrapped into the nano-scale fibers around the chambers similar to the situation in liposome or SLNs. This could be the mechanism of the protective effect of the supramolecular gel on the photostability of RP.

To further confirm the suggestion that RP is located in the "chambers", we applied fluorescent optical microscopy (FOM) because RP is fluorescent-active. Figure 6 shows the
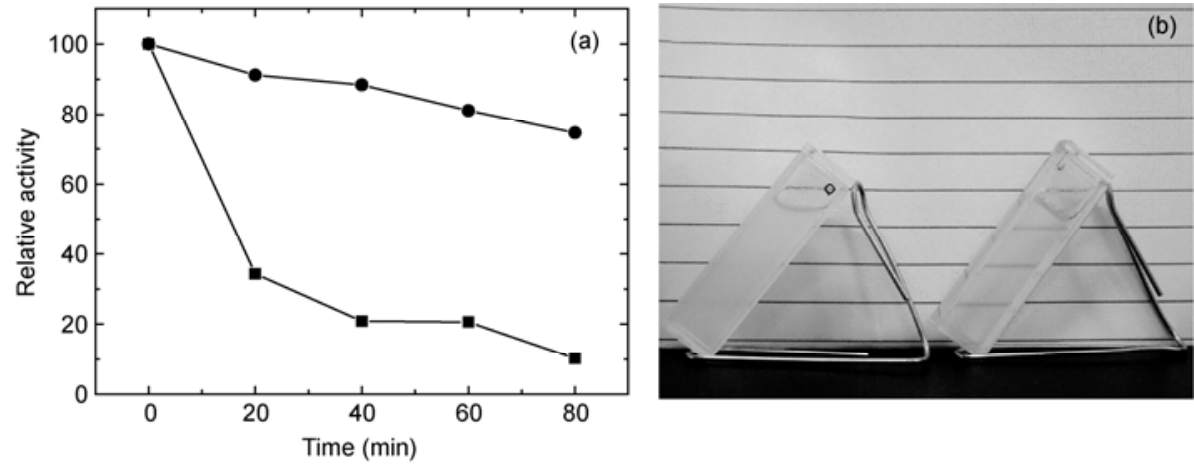

Figure 4 (a) Photostability of RP in HEC gels ( $)$ and in MDBS gels (•). The HEC gel is formed from 2 wt $\%$ of HEC and 98 wt $\%$ of water. The MDBS gel was formed from PEG400/ethanol $(1 / 2, \mathrm{v} / \mathrm{v})$ in the presence of $0.6 \mathrm{wt} \%$ of MDBS. The concentration of RP in all samples was $3.81 \times 10^{-3} \mathrm{~mol} / \mathrm{L}$. (b) Photograph: HEC gel (left) and MDBS gel (right). 

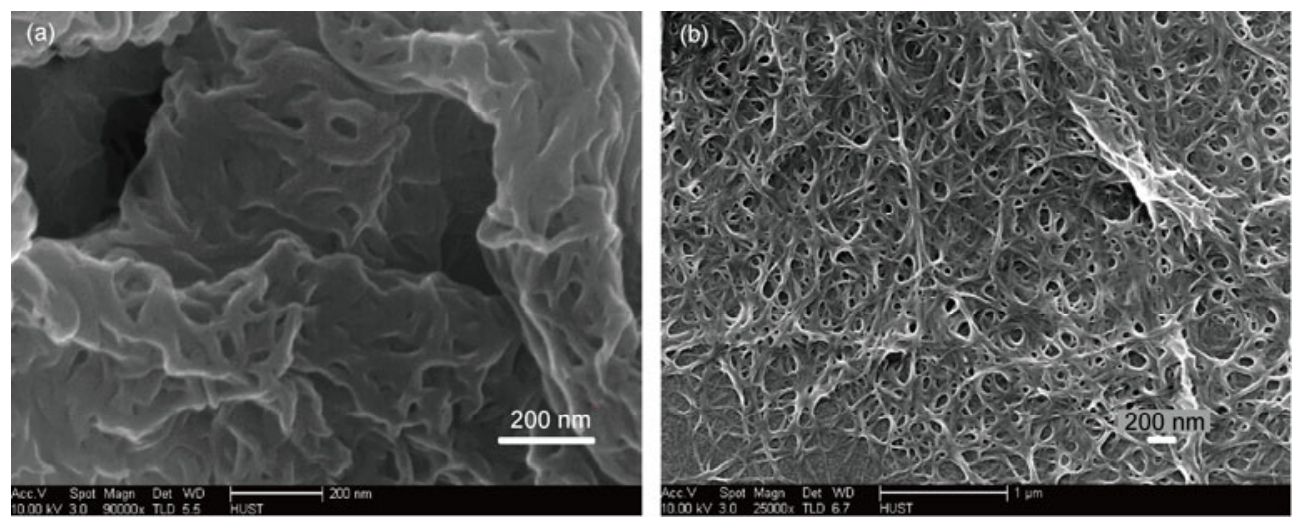

Figure 5 FE-SEM images of MDBS xerogels (propylene glycol/ethanol, 1/2, v/v). (a) MDBS gel without RP, (b) MDBS gel with RP. The concentration of MDBS in all samples is $0.8 \mathrm{wt} \%$, the concentration of RP in all samples is $2.5 \times 10^{-3} \mathrm{~mol} / \mathrm{L}$.
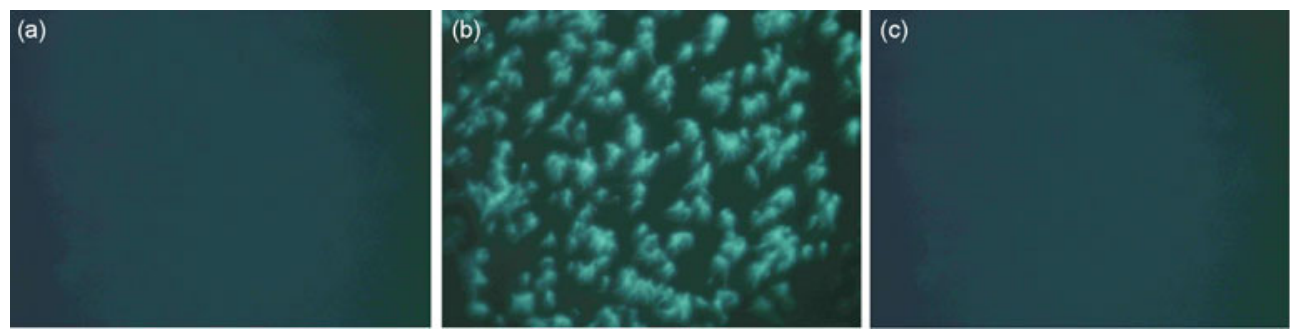

Figure 6 FOM images of (a) PEG400/ ethanol solution (1/2, v/v) containing RP, (b) MDBS gels with RP and (c) MDBS gels without RP. The concentration of MDBS and RP is $0.4 \mathrm{wt} \%$ and $6 \times 10^{-4} \mathrm{~mol} / \mathrm{L}$, respectively. The magnification of the images is $400 \times$.

FOM images of RP in MDBS gels and in the corresponding solution. Obviously, almost no luminescence is observed in the case of the solution (Figure 6(a)), indicating that RP is homogeneously dispersed in the solution. However, significantly enhanced luminescence is observed in the gel. RP appears as dispersive light spots in Figure 6(b). In a control experiment, no luminescence was observed in MDBS gels without RP (Figure 6(c)). The results reveal that RP molecules reside in the chambers of three-dimensional networks. Since the degradation process of RP is a chain reaction [1], the isolation of RP in the chambers plays an important role to reduce the probability of collision of the photoactivated RP molecules [5]. This may be the main reason for the protective effect of the supramolecular gels on the photostability of RP.

\subsection{UV spectra of RP in MDBS gels and the corre- sponding solutions}

The above discussion indicates that the retained activity of $\mathrm{RP}$ entrapped in supramolecular gels is significantly improved in comparison with that in the corresponding solutions. Now a relevant question is whether the chemical structure and properties of RP are changed by the interactions between RP and the microenvironment? Thus, we determined the UV spectra of RP in MDBS gels and in the corresponding solution (Figure 7). The data indicate that both UV spectra are almost the same. We conclude that,

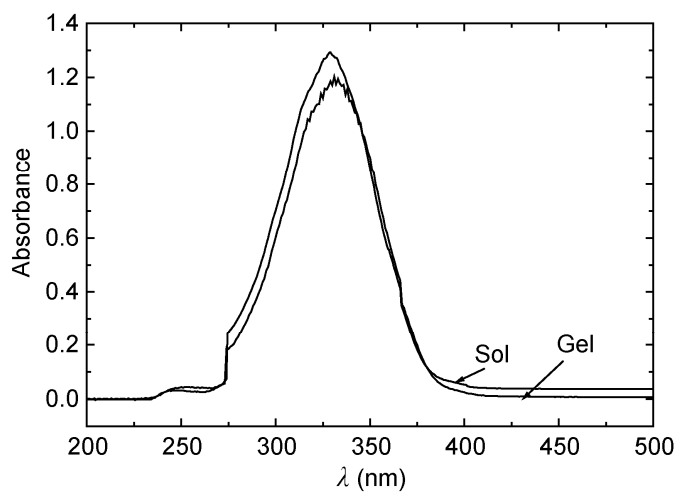

Figure 7 UV spectra of RP in MDBS gels and in the corresponding solution (PEG400/ethanol, 1/2, v/v). Concentrations of MDBS and RP were 0.4 wt $\%$ and $2 \times 10^{-5} \mathrm{~mol} / \mathrm{L}$, respectively.

although the microenvironments of RP located in the gels and the solutions are different, the chemical structure of RP has not changed. RP is only physically entrapped into the gel and its chemical and physiological activity is not affected.

\section{Conclusions}

In this work, we propose a new strategy for protecting the photostability of RP. Supramolecular gels were prepared by self-assembly of a series of sorbitol-based gelators in a 
solvent mixture of PEG400/ethanol or 1,2-propylene glycol/ethanol. MDBS gels exhibited excellent protective effects on the photostability of RP. In comparison with conventional polymeric HEC gels, supramolecular gels significantly increase the retained activity of RP after irradiation of UVA. The mechanism of the protective effect may involve the particular microenvironment of RP located in the MDBS gel and the microstructure of the MDBS gel. The data show that RP is located and isolated into the solvent chambers formed by the three-dimensional network structure of the gel.

We thank the Analytical Test Center of Huazhong University of Science and Technology for all measurements. This work was supported by the National Natural Science Foundation of China (51073062) and the National Basic Research Program of China (2012CB812500).

1 Fu P P, Xia Q, Yin J J, et al. Photodecomposition of Vitamin A and photobiological implications for the skin. Photochem Photobiol, 2007, 83: 409-424

2 Eskandar N G, Simovic S, Prestidge C A. Chemical stability and phase distribution of all-trans-retinol in nanoparticle-coated emulsions. Int J Pharm, 2009, 376: 186-194

3 Loveday S M, Singh H. Recent advances in technologies for Vitamin A protection in foods. Trends Food Sci Technol, 2008, 19: 657-668

4 Manabu H, Tomoya O, Akito O, et al. Vitamin A-containing ophthalmic compositions method for Vitamin A stabilization using polyoxyethylene-polyoxypropylene glycols. Japanese Patent, 2009173638

5 Semenzato A, Bau A, Dallaglio C, et al. Stability of Vitamin A palmitate in cosmetic emulsions: Influence of physical parameters. J Cosmet Sci, 2007, 16: 139-147

6 Young A M, Gregoriadis G. Photolysis of retinol in liposomes and its protection with tocopherol and oxybenzone. Photochem Photobiol, 1996, 63: 344-352

7 Carlotti M E, Sapino S, Trotta M, et al. Photostability and stability over time of retinyl palmitate in an O/W emulsion and in SLN introduced in the mulsion. J Dispersion Sci Technol, 2005, 26: 125-138

8 Carlotti M E, Rossatto V, Gallatate M, et al. Vitamin A palmitate potostability and stability over time. J Cosmet Sci, 2004, 55: 233-252

9 Sapino S, Carlotti M E, Cavalli R, et al. Effect of akyl- $\gamma$-cyclo- dextrins on the stability of retinol. J Incl Phenom Macrocycl Chem, 2007, 57: 451-455

10 Terech P, Weiss R G. Low molecular mass gelators of organic liquids and the properties of their gels. Chem Rev, 1997, 97: 3133-3159

11 George M, Weiss R G. Molecular organogels. Soft matter comprised of low-molecular-mass organic gelators and organic liquids. Acc Chem Res, 2006, 39: 489-497

12 Banerjee S, Das R K, Maitra U. Supramolecular gels “in action”. J Mater Chem, 2009, 19: 6649-6687

13 Miyata T, Uragami T, Nakamae K. Biomolecule-sensitive hydrogels. Adv Drug Deliv Rev, 2002, 54: 79-98

14 Mohmeyer N, Wang P, Schmidt H, et al. Quasi-solid-state dye sensitized solar cells with 1,3:2,4-di- $O$-benzylidene- $D$-sorbitol derivatives as low molecular weight organic gelators. J Mater Chem, 2004, 14: 1905-1909

15 Watase M, Itagaki H. Thermal and rheological properties of physical gels formed from benzyllidene-D-sorbitol derivatives. Bull Chem Soc Jpn, 1998, 71: 1457-1466

16 Watase M, Nakatani Y, Itagaki H. On the origin of the formation and stability of physical gels of di- $O$-benzylidene- $D$-sorbitol. J Phys Chem B, 1999, 103: 2366-2373

17 Wang H, Zhang W, Dong X, et al. Thermo-reversibility of the fluorescence enhancement of acridine orange induced by supramolecular self-assembly. Talanta, 2009, 77: 1864-1868

18 Wang H, Zhang J, Zhang W, et al. Host-guest interactions of 5fluorouracil in supramolecular organogels. Eur J Pharm Biopharm, 2009, 73: 357-360

19 Friggeri A, Feringa B L, van Esch J. Entrapment and release of quinoline derivatives using a hydrogel of a low molecular weight gelator. J Control Release, 2004, 97: 241-248

20 Bastiat G, Leroux J. Pharmaceutical organogels prepared from aromatic amino acid derivatives. J Mater Chem, 2009, 19: 867-877

21 Sutton S, Campbell N, Cooper A I, et al. Controlled release from modified amino acid hydrogels governed by molecular size or network dynamics. Langmuir, 2009, 25: 10285-10291

22 Wilder E A, Hall C K, Khan S A, et al. Effects of composition and matrix polarity on network development in organogels of poly (ethylene glycol) and dibenzylidene sorbitol. Langmuir, 2003, 19: 6004-6013

23 Zhu G, Dordick J S. Solvent effect on organogel formation by low molecular weight molecules. Chem Mater, 2006, 18: 5988-5995

24 Inamura I, Isshiki M, Aroki T. Solubilization of hemin in neutral and acidic aqueous solutions by forming complexes with water-soluble macromolecules. Bull Chem Soc Jpn, 1989, 62: 2413-2415

Open Access This article is distributed under the terms of the Creative Commons Attribution License which permits any use, distribution, and reproduction in any medium, provided the original author(s) and source are credited. 